\title{
Le \\ recyclage de l'eau dans la papeterie
}

PAR

D. Gros

Ingénieur I.N.S.C.I.R., Agence Financière de Bassin

Rhin-Meuse

Il existe environ vingt papeteries dans le Bassin RhinMeuse. Sur ce nombre, quinze ont entrepris ou mené à bien un programme de lutte contre la pollution des eaux ayant donné lieu à une intervention de l'Agence de Bassin. $\mathrm{Ce}$ résultat, obtenu en quatre ans grâce à une politique systématique d'aides techniques et d'incitations financières, permet de dégager certaines règles générales de luttte contre la pollution dans une profession dont les productions sont relativement diverses.

Il n'est pas question de passer en revue, ici, l'ensemble des problèmes que l'on peut rencontrer en ce domaine, ni de décrire toutes les techniques employées, mais plutôt de donner quelques principes généraux appuyés de chiffres, et de les étayer par un exemple détaillé, celui de la Cartonnerie Béghin de Kaysersberg.

\section{Principes des actions de réduction de la pollution}

On sait que de nombreuses possibilités existent d'opérations internes, permettant d'économiser l'eau, notamment par recyclage.

Ces opérations conduisent souvent à des économies qui devraient amener l'industriel avisé à les réaliser. Les limites fixées par la seule recherche de la rentabilité résulteraient essentiellement :

- de la capacité đu papier à recueillir des fibres de récupération, sans altérer ses qualités;
- de la fréquence des changements de fabrication;

- des paramètres de fabrication (égouttage, vitesse de machine, etc.) qui sont réglés pour une certaine qualité de fibres.

Cependant, l'intérêt de ces opérations internes apparaît encore plus grand dans une optique de lutte contre la pollution des eaux. En effet, elles permettent de diminuer la quantité d'eaux résiduaires et la charge contenue dans ces eaux.

Si une station d'épuration est nécessaire, son coût est moindre; dans certains cas de récupérations poussées à fond, on attendra le stade du circuit fermé ou un flux de pollution suffisamment bas pour que le milieu naturel laccepte sans épuration. Dans tous les cas, la nécessité de lutte contre la pollution poussera lindustriel avisé à mener les opérations internes de recyclage au-delà de ce que dicte la rentabilité immédiate.

Les quinze programmes de lutte contre la pollution, menés dans des papeteries du Bassin Rhin-Meuse, font appel à une phase, la plupart du temps la première, qui consiste à réorganiser les réseaux d'eau et d'égout et à mettre en place un recyclage, le plus poussé possible.

Dans certains cas, et c'est notamment vrai pour les cartonneries, c'est la station elle-même qui sert de récupérateur de pâte global pour toute l'usine. Les boues sont retournées au pulper, tandis que l'eau, généralement après un traitement de finition, est réutilisée dans l'usine.

Dans d'autres cas, il s'agit alors de papeteries fabriquant du papier impression-écriture, la récupération et le recyclage sont poussés le plus loin possible, puis les eaux résiduaires restantes sont traitées dans une station d'épuration avant d'être rejetées à la rivière. 


\section{Les mesures internes}

Les papeteries sont très différentes les unes des autres et chaque usine est un cas particulier, fonction non seulement du type de papier fabriqué, mais encore de l'âge de l'usine, de la place disponible, etc. Dans chaque cas, la conduite à tenir sera modulée par ces éléments, mais quel que soit le type d'usine, les mesures internes de la lutte contre la pollution concerneront simultanément :

- les économies d'eau;

- la récupération des «fines» (cellulose, charges) autrefois perdues;

ces deux types d'action étant d'ailleurs indissociables.

\section{1 - SÉparation des RÉseaux}

La séparation des circuits présente un avantage essentiel qui apparaît pleinement quand on passe à l'épuration externe. En effet, le coût de la dépollution finale à la sortie de l'usine dépend surtout du débit des eaux résiduaires, et beaucoup moins de la charge qu'elles contiennent. C'est le cas notamment pour les décanteurs dont les dimensions sont fixées uniquement par le débit entrant.

Il est essentiel d'éviter de mélanger aux eaux résiduaires chargées, les eaux claires qui peuvent être, soit rejetées directement, soit réutilisées en l'état. Il faut donc un double réseau, ou réseau séparatif.

Avec les eaux claires, doivent s'écouler :

- les eaux de refroidissement de sécherie;

- les eaux des pompes à anneau liquide lorsqu'elles sont claires;

- les eaux de purge et de refroidissement de la chaudière.

\section{2 - LES ÉCONOMIES PROPREMENT dITES}

Avant tout recyclage, il convient d'éviter le simple gâchis. Par exemple, il est fréquent, dans les papeteries, de trouver les eaux de laveurs manuels (ou «pisseuses») grandes ouvertes et s'écoulant directement dans le caniveau : chaque pisseuse peut représenter $3 \mathrm{~m}^{3} / \mathrm{h}$. Il est simple pourtant, soit de fermer les vannes quand elles ne servent pas, soit d'équiper les pisseuses de vannes-pistolets.

Cet exemple permet d'illustrer un état d'esprit : jusqu'ì présent, le papetier se souciait relativement peu de sa consommation en eau et, chez lui, il valait mieux qu'une vanne soit ouverte que fermée, surtout quand il s'agissait d'eau de refroidissement sans conséquence directe sur la fabrication du papier. Cet état d'esprit est incompatible avec l'efficacité de la lutte contre la pollution.

Autre exemple significatif : on peut citer des usines dont une partie importante de la «consommation» d'eau provenait directement du trop-plein du château d'eau.

On traitait, à grands frais, de l'eau de rivière dont on laissait partir dans les eaux résiduaires une fraction notable, faute d'avoir installé une régulation sur la pompe d'alimentation. Dans les mêmes conditions, une épuration des eaux résiduaires aurait été fortement sur-dimensionnée.

Soulignons que si la lutte contre la pollution passe d'abord par une volonté de réduire les consommations inutiles dans l'usine, cette volonté peut difficilement aboutir sans qu'elle soit partagée par tout le personnel. En tout état de cause, l'installation de compteurs d'eau est un premier pas indispensable.

\section{3 - LES RECIRCULATIONS D'EAU DANS L'USINE}

Traditionnellement, les papeteries ont recyclé les eaux blanches sous la toile pour récupérer les grandes quantités de fibres et de charges qui s'y trouvent.

Les eaux sous les pontuseaux, qui sont les plus chargées, sont réutilisées directement juste avant la caisse de tête.

Les autres eaux blanches, moins chargées que les précédentes, servent à alimenter le pulper (cf. $\$ 1-1$ ), et opérer toutes les dilutions successives jusqu'à la caisse de tête. Mais toutes ces utilisations ne suffisent pas à consommer les grosses quantités d'eau disponibles sous la machine à papier.

Il y a donc en général, un excès d'eau blanche.

Deux cas peuvent se produire:

- Ou bien l'usine travaille avec une matière première «noble» (pâte kraft par exemple), et ne change pas trop souvent de qualité de papier. Dans ce cas, l'industricl a intérêt à installer un récupérateur de pâte qui sépare la fibre récupérable de l'eau. Il fait des économies de pâte et en même temps il régénère une eau qui peut être recycléc en partie sur les rinceurs de toile par exemple.

La plupart des usines où l'on produit des qualités classiques sont déjà équipées ainsi.

- Ou bien l'usine travaille avec des matières premières très bon marché (vieux papiers), ou change très souvent dc fabrication ou de couleur, ou encore fait des papiers très chargés ou couchés.

Même dans ce cas, l'industriel a intérêt à s'équiper en ramasse-pâte et à recycler; l'intérêt provient alors moins de la valeur des produits qu'il récupère que des économies de pollution qu'il réalise, et qui lui permettent ultérieurement de diminuer la taille de sa station d'épuration' ou même, dans certains cas exceptionnels, de s'en dispenser.

Un exposé a déjà été fait devant vous sur les procédés employés pour le recyclage de l'eau en papeterie, et je n'y reviendrai pas. Je me contenterai de donner ci-dessous un exemple succinct d'une papeterie impression-écriture ayant mené à bien un programme de recyclage de ses eaux, et un exemple plus détaillé d'une cartonnerie qui essaie de passer en circuit fermé.

\section{Papeterie Metenett (Raon-l'Etape)}

\section{RÉALISATION EN 1974}

- Fabrication : impression-écriture et emballage : $70 \mathrm{t} / \mathrm{j}$.

- Consommation spécifique et pollution à la tonne de papier produit :

$\begin{array}{lrr}\text { - avant transformation. } 90 \mathrm{~m}^{3} / \mathrm{t} & 44,1 \mathrm{~kg} \mathrm{MES} / \mathrm{t} \\ \text { - après transformation. } & 37 \mathrm{~m}^{3} / \mathrm{t} & 4,7 \mathrm{kgMES} / \mathrm{t} \\ \text { (filtre Vargo) } & & \end{array}$

Avant rejet, l'eau est décantée dans deux bassins de 3000 et $5000 \mathrm{~m}^{3}$ chacun, assurant une pollution spécifique de moins de $1 \mathrm{~kg}$ de MES à la tonne fabriquée. 


\section{L'usine de Kaysersberg de la Société Beghin S.A.}

La Cartonnerie de Kaysersberg fabrique 200 tonnes par jour de cartons de différentes qualités, soit environ $65000 \mathrm{t} / \mathrm{an}$. Elle est située sur une petite rivière, la Weiss, dont le débit réduit a toujours nécessité une politique sérieuse de lutte contre la pollution.

En 1972, l'usine consommait $620 \mathrm{~m}^{3}$ d'eau à l'heure, et rejetait la plus grosse partie de cette eau à travers un décanteur Degrémont assez ancien (1953) dans la rivière. L'eau contenait encore plus de $50 \mathrm{mg} / 1$ de MES et $150 \mathrm{mg} / \mathrm{l}$ de $\mathrm{DCO}$, et parfois beaucoup plus quand il y avait des incidents de marche (l'eau à l'entrée du décanteur étant chargée à $1000 \mathrm{mg} / \mathrm{l}$ de MES environ). De plus, d'énormes difficultés étaient rencontrées dans le recyclage des boues décantées par suite de bouchage, si bien que souvent les boues n'étaient pas recyclées et qu'il était nécessaire de vidanger entièrement le bassin, avec les conséquences que l'on imagine pour la rivière.

La Société Béghin a réalisé, avec l'aide de l'Agence et du FIANE, une modification complète de son installation d'épuration, qui a eu pour conséquences :

- d'épurer l'eau;

- de la recycler en fabrication;

- de réutiliser les boues.

La réalisation a été conduite en trois étapes :

\section{1 - AMÉlIORATION \\ DU DÉCANTEUR FLOCULATEUR}

Le bassin raclé, cylindro-conique de $27 \mathrm{~m}$ de diamètre, assure une vitesse ascensionnelle de 1 à $1,5 \mathrm{~m} / \mathrm{h}$. Le système d'évacuation des boues a été modifié et remplacé par un puits de pompage muni de deux groupes immergés de $60 \mathrm{~m}^{3} / \mathrm{h}$ chacun. Cette modification a permis d'éviter les bouchages, donc les arrêts de décantation, de mieux floculer en obtenant une boue plus concentrée et, enfin, de produire une eau mieux décantée (au moins $35 \mathrm{mg} / \mathrm{l}$ de moyenne mensuelle) et moins chargée en matières oxydables du fait de l'adsorption du floc. Production de boues: $12 \mathfrak{t} /$ jour diluées dans 30 fois leur poids d'eau.

\section{2 - MODIFICATION DES CIRCUITS DE BOUES ET D'EAUX}

Les boues sont soutirées du décanteur et envoyées dans un bassin intermédiaire avant d'être pompées vers les différentes utilisations en fabrication. Soit directement au niveau des pulpers, soit après tamisage et cyclonage vers un épaississeur ou une centrifugeuse. Après la centrifugeuse, les boues passent dans un appareil Asplund où elles peuvent être stockées, déshydratées, sans risque de les voir fermenter avant leur réutilisation. Ces boues, en effet, sont réutilisées dans les couches intérieures d'un carton qui en comportc jusqu'à sept. Les filtrats et les centrats sont retournés en tête de bassin.

\section{3 - MODIFICATION DU CIRCUIT DES EAUX BLANCHES}

Il a été prévu dès le départ, de tendre vers une mise en circuit fermé de l'eau en mettant en œuvre:

- une diminution de la consommation d'eau;

- un traitement des eaux en vue de leur réutilisation.

\section{Diminution de la consommation d'eau}

Chacune des deux machines à carton comporte quatre circuits indépendants d'eau et de pâte. Sur ces circuits, quatre étaient complètement fermés, c'est-à-dire que toute l'eau provenant de la mise en feuille est récupérée et réutilisée pour la mise en pâte; il a fallu fermer les quatre autres pour supprimer l'utilisation d'eau claire à la mise en pâte.

Les rinceurs de toiles des formes rondes ont été remplacés par des rinceurs à eau blanche.

Le lavage des feutres a été assuré par des rinceurs haute pression.

L'eau ayant servi au refroidissement a été injectée dans le circuit de fabrication. 2

\section{Réutilisation des eaux usées}

Escher Wyss a réalisé, à la sortie du décanteur, une installation de filtration destinée à traiter $400 \mathrm{~m}^{3} / \mathrm{h}$ d'eau à $30 \mathrm{mg} / 1$ de MES. Il s'agit d'un bassin d'homogénéisation et

\section{Tableau 1}

\begin{tabular}{|c|c|c|c|c|c|c|c|c|}
\hline & $\begin{array}{c}\text { Productron } \\
(\mathrm{t} / \mathrm{h})\end{array}$ & $\begin{array}{l}\text { DÉBIT } \\
\text { REJETÉ } \\
\left(\mathrm{m}^{3} / \mathrm{h}\right)\end{array}$ & $\begin{array}{c}\text { MES } \\
\text { ENTRÉE } \\
\text { DÉCANTEUR } \\
(\mathrm{mg} / \mathrm{l})\end{array}$ & $\begin{array}{c}\text { MES } \\
\text { SORTIE } \\
\text { DÉCANTEUR } \\
(\mathrm{mg} / \mathrm{l})\end{array}$ & $\begin{array}{c}\text { MES } \\
\text { SORTIE } \\
\text { FILTRES } \\
(\mathrm{kg} / \mathrm{j})\end{array}$ & $\begin{array}{c}\text { DBO } \\
\text { SORTIE } \\
\text { EFFLUENT } \\
(\mathrm{mg} / \mathrm{l})\end{array}$ & $\begin{array}{c}\text { DCO } \\
\text { SORTIE } \\
\text { EFFLUENT } \\
(\mathrm{mg} / \mathrm{l})\end{array}$ & $\begin{array}{l}\text { MOX } \\
(\mathrm{mg} / 1)\end{array}$ \\
\hline $\begin{array}{l}1970 \ldots \ldots \ldots \ldots \\
1971 \ldots \ldots \ldots \ldots \\
1972 \ldots \ldots \ldots \\
1973 \ldots \ldots \ldots \ldots \\
1974 \text { (10 mois) } \ldots \ldots\end{array}$ & $\begin{array}{l}6,4 \\
6,8 \\
7,2 \\
7,2 \\
8,3\end{array}$ & $\begin{array}{l}570 \\
597 \\
576 \\
479 \\
425\end{array}$ & $\begin{array}{l}460 \\
528 \\
625 \\
711 \\
980\end{array}$ & $\begin{array}{l}37 \\
38 \\
38 \\
31 \\
35\end{array}$ & & $\begin{array}{l}60 \\
53,5 \\
69 \\
69 \\
72\end{array}$ & $\begin{array}{l}174 \\
170 \\
188 \\
189 \\
245\end{array}$ & \\
\hline $\begin{array}{l}\text { Novembre } 1974 \ldots \ldots \\
\text { Décembre } 1974 \ldots \ldots\end{array}$ & $\begin{array}{l}8,4 \\
7,5\end{array}$ & $\begin{array}{l}155 \\
145\end{array}$ & $\begin{array}{l}1093 \\
1198\end{array}$ & $\begin{array}{l}23 \\
34\end{array}$ & $\begin{array}{l}9,6 \\
20\end{array}$ & $\begin{array}{l}63 \\
90\end{array}$ & $\begin{array}{l}410 \\
533\end{array}$ & \\
\hline
\end{tabular}


Tableau 2

\begin{tabular}{|c|c|c|c|c|c|c|c|}
\hline & $\begin{array}{c}\text { PrODUCTION } \\
(\mathrm{t} / \mathrm{h}) \\
\end{array}$ & $\begin{array}{c}\text { EAU POLLUÉE } \\
\text { REJETEE } \\
\left(\mathrm{m}^{3} / \mathrm{t}\right) \\
\end{array}$ & $\begin{array}{c}\text { MES } \\
\text { SORTIE } \\
\text { EFFLUENT } \\
(\mathrm{kg} / \mathrm{t}) \\
\end{array}$ & $\begin{array}{c}\text { DBO } \\
\text { SORTIE } \\
\text { EFFLUENT } \\
(\mathrm{kg} / \mathrm{t}) \\
\end{array}$ & $\begin{array}{c}\text { DCO } \\
\text { SORTIE } \\
\text { EFFLUENT } \\
(\mathrm{kg} / \mathrm{t}) \\
\end{array}$ & $\begin{array}{r}\text { MES } \\
(\mathrm{kg} / \mathrm{j}) \\
\end{array}$ & $\begin{array}{l}\text { MOX } \\
(\mathrm{kg} / \mathrm{j}) \\
\end{array}$ \\
\hline $1970 \ldots \ldots \ldots \ldots$ & 6,4 & 89 & 3,30 & 5,3 & 15,5 & 506 & 1,339 \\
\hline $1971 \ldots \ldots \ldots \ldots \ldots$ & 6,8 & 88 & 3,35 & 4,7 & 15 & 546 & 1321 \\
\hline $1972 \ldots \ldots \ldots \ldots \ldots$ & 7,2 & 80 & 3,30 & 5,5 & 15 & 570 & 1506 \\
\hline $1973 \ldots \ldots \ldots \ldots \ldots$ & 7,2 & 60,5 & 2,1 & 4,6 & 12,6 & 362 & 1158 \\
\hline 1974 (10 mois) ..... & 8,3 & 51 & 1,8 & 3,7 & 12,6 & 358 & 1320 \\
\hline Novembre 1974 & 8,4 & 18,5 & 0,18 & 1,16 & 7,5 & 36 & 664 \\
\hline Décembre 1974 & 7,5 & 19,4 & 0,38 & 1,73 & 10,2 & 52 & 819 \\
\hline
\end{tabular}

de trois filtres à sable, dont le nettoyage peut se faire à tour de rôle à contre-courant.

L'eau sort des filtres avec une charge comprise entre 5 et $10 \mathrm{mg} / 1$ de MES, elle passe ensuite par une installation de chloration avant d'être, soit recyclée vers l'usine, soit rejetée pour une part à la rivière.

\section{Les résultats}

Sur le plan des résultats, la mise en circuit fermé a fonctionné deux mois en 1974 (novembre et décembre), mais avec un taux de recyclage inférieur à celui qui était prévu, et donc avec des charges polluantes supérieures aux prévisions. Le tableau 1 résume l'évolution constatée.

Ces chiffres peuvent également se lire en valeurs spécifques, c'est-à-dire ramenés à la tonne de carton produite (tableau 2).

Les résultats sont spectaculaires, puisque aussi bien sur le plan de la pollution spécifique en MES que sur celui de la pollution spécifique organique, ils sont très inférieurs au programme de branche : respectivement $2 \mathrm{~kg}$ de $\mathrm{MES} / \mathrm{t}$ et $2 \mathrm{~kg}$ de $\mathrm{DBO} / \mathrm{t}$, mais ils ne sont pas à la hauteur des espérances et des chiffres que la convention avait prévus :

1. Teneur des eaux recyclées de 10 à 20 ppm en MES : objectif atteint.

2. Homogénéisation, régulation de $\mathrm{pH}$, chloration : objectif atteint.

3. Recyclage en fabrication de l'ordre de $90 \%$ de volume : le degré de recyclage n'est que de $66 \%$ environ, la purge se faisant comme prévu sur eau filtrée à moins de $20 \mathrm{ppm}$ en MEST.

4. Rejet de $20 \mathrm{~kg} / \mathrm{j}$ de MEST et de $65 \mathrm{~kg} / \mathrm{j}$ de MOX : ces objectifs fixés dans le contrat du FIANE, sont loin d'être atteints, en particulier pour les MOX. Il est à remarquer que ces derniers objectifs ont été fixés en valeur absolue et non en valeur spécifique, ce qui les rend d'autant plus difficiles à atteindre que la production journalière a augmenté de $20 \%$ environ depuis 1970.

Les chiffres cités dans les tableaux ci-dessus minimisent relativement les performances de l'installation, car ce sont des moyennes mensuelles qui intègrent les pannes et incidents qui ont pu intervenir dans les mois en question.

On sait que, généralement, les performances sont données pour un ou plusieurs jours de marche normale, c'est-à-dire sans incidents.

Cependant, les résultats obtenus sont encore insuffisants et peuvent être améliorés. En particulier, il est possible raisonnablement de demander à l'usine de diminuer encore son rejet, par exemple à moins de $9 \mathrm{~m}^{3} / \mathrm{t}$ soit $72 \mathrm{~m}^{3} / \mathrm{h}$ pour une production de $8 \mathrm{t} / \mathrm{h}$ (chiffre à comparer aux $600 \mathrm{~m}^{3} / \mathrm{h}$ du départ). Il est possible également de descendre à $20 \mathrm{~kg}$ de MES/jour, ce qui fait un rejet de $100 \mathrm{~g}$ de MEST par tonne de papier fabriqué.

Ces chiffres pourront être obtenus par certaines modifications en cours de réalisation dans les circuits des machines :

- fermeture complète du circuit de tête de machine de la table plate de la machine 2 ;

- réutilisation des eaux des pompes à vide pour les rinceurs des rouleaux de la table plate;

- alimentation des rinceurs de bords de feuille en eau recyclée;

- passage des derniers rinceurs de la machine 1 en eau recyclée.

Une amélioration du fonctionnement des filtres à sable peut être également obtenue.

\section{Coût des investissements et aides}

Le coût total des investissements s'élève à $2840000 \mathrm{~F}$. Sur ce montant, la Société Béghin a reçu, soit de l'Agence de Bassin, soit du FIANE, $1232100 \mathrm{~F}$ sous forme de subvention, $165000 \mathrm{~F}$ sous forme d'avance sans intérêt sur douze ans et $455000 \mathrm{~F}$ sous forme de prêt sur douze ans. 


\section{Discussion}

Président : M. D. MOYEN

Je remercie M. DANiel de son exposé fort documenté sur L'épuration des eaux résiduaires des charbonnages: possibilités de recyclage. qui éroque un cas précis : celui de la rivière La Roselle.

Votre rapport ouvre deux voies: l'une, en filigrane, consisterait dans la fermeture des usines qui engendrent la pollution - solution radicale qui ne nous intéresse pas beaucoup - l'autre, est celle du recyclage de l'eau, non plus dans l'usine elle-même, mais par un usage «croisé» de celle-ci entre les houillères et la chimie (en profitant d'une certaine complémentarité des besoins). On y reviendra peut-être tout à l'heure. Mais je préférerais quauparavant, M. Gros vienne, en une demi-heure, nous exposer le problème des papeteries

M. Gros présente sa communication intitulée: Le recyclage do l'eau dans les papeteries.

\section{象}

M. Marécaux (Sté Spéchim) demande quelle est l'utilisation des schlamms déposés dans les bassins.

Ces schlamms n'ont pour l'instant aucune utilisation, répond M. DANIEl, mais la zone où se trouvent les carrières aménagées en bassin de décantation étant très urbanisée, on peut espérer qu'après leur comblement par les dépôts, ces bassins seront aménagés en espaces verts.

Les cuvettes réalisées étaient, à l'origine, profondes de 30 à $35 \mathrm{~m}$; mais elles ne peuvent s'emplir entièrement d'eau car la tour do reprise comporte des déversoirs réglables qui maintiennent, en permanence, une couche d'eau de 2 ou $3 \mathrm{~m}$ d'épaisseur seulement au-dessus de la surface des matériaux déposés.

M. le Professeur Carta pose les deux questions ci-après :

$1^{\circ}$ Quel est le $\mathrm{pH}$ des eaux de la Roselle?

$2^{\circ}$ Comment a été réalisée l'étanchéité du fond de la carrière ?

M. DANiel répond:

$1^{\circ}$ Le pH des eaux de la Roselle est compris entre 7,8 et 8 .

$2^{\circ}$ Pour deux de nos carrières, le problème ne s'est pas posé. On n'était pas dans une zone de captage d'eau et, d'autre part, les eaux de ces deux sièges contenaient de l'ordre de $200 \mathrm{mg}$ de chlorures par litre; on ne s'est donc pas préoccupé, pour ces deux sièges, d'assurer l'élanchéité du fond. Pour l'autre bassin, celui de Merlebach, du fait de la très forte concentration en chlorures et de l'existence, dans la grande zone d'influence de cette carrière, de captages d'eau potable, il a fallu la rendre étanche. A cet effet un film plastique a été posé sur lit de sable au fond de la carrière avec une remontée sur $3 \mathrm{~m}$ tout autour. Ainsi, l'étanchéité du fond est assurée pendant la période où il n'y aura pas une couche de schlamms suffisante pour la réaliser. Si, dans deux ou trois ani, le film plastique s'est détérioré (notamment du fait de sa mauvaise tenue mécanique) il y aura de toute manière une couche d'eau d'au moins $1,50 \mathrm{~m}$ à $2 \mathrm{~m}$ de schlamms déposés, ce qui devrait assurer une étanchéité très largement suffisante.

M. le Président observe que l'on a abordé trop rapidement la question du coút des méthodes de recyclage et de lutte contre ja pollution. Il est d'alleurs généralement difficile de discriminer ta part des investissements et les dépenses d'exploitation entraînés par ces dernières et celle dûe à une fabrication jugée jusqu'ici «normale ».

Si l'on voulait, dit-il, calculer le coût pour les usines Renault et pour le public des nuisances dues au bruit, faudrait-il prendre eil compte, pour chaque voiture, le coût de chaque pot d'échappemeni et le prix des bourrelets de porte qui permettent de diminuer les bruits?

Comment procède-t-on, dans les Agences de Bassin, pour distinguer parmi les investissements de recyclage et d'anti-pollution, ceux qui sont rentables pour l'industriel et ceux qui ne le sont pas?

M. DANIEL répond:

Nous avons effectivement buté sur cette difficulté. Un industrie: nous avait présenté un projet astucieux touchant, en fait, au pro. cessus de fabrication mais ayant une action importante et bénéfiquc sur la lutte contre la pollution. Nous avons tenté de diviser chaque élément de ce projet en deux: une partie «anti-pollution » et unc partie "production »; cela s"est révélé très difficile. On a donc essayé de mettre au point une formule permettant d'estimer la rentabilité partielle on totale - positive ou négative - de certains investissements. La formule que nous avons retenue est actuellement en discussion dans les Agences de Bassin afin que tous les industriels soient traités, à ce point de vue, sur un pied d'égalité. Voici cetle formule :

$$
I_{s}=0,67 I-5,56 i
$$

$I$. est l'investissement retenu par l'Agence de Bassin;

$I$ est l'investissement présenté par l'industriel sur son projet;

$i$ est le taux de rentabilité annuelle pour l'industriel de celto réalisation.

Si l'investissement est entièrement rentable, on admet que : $i=12 \%$ de $I$; il s'agit dans ce cas d'un investissement de production et l'Agence de Bassin n'a pas à intervenir; c'est là un point de vue que ne partagent pas toujours les industriels. Dans le cas extrême d'une station d'épuration qui n'a aucune rentabilité propre pour lindustriel et qui, même, lui coûte des frais d'exploitation : $i=-6 \%$ de $I$.

Il était important, observe $M$. le Président, d'examiner cette question. Les exposés de ce matin ont toujours cherché un équilibre entre l'économie pour l'industriel, l'économie d'eau et la lutte contre la pollution; ce sont trois données, trois objectifs qui coexistent et qui ne sont pas forcément concomitants; ils le sont de temps en temps, et lorsqu'il faut envisager une intervention financière, il est difficile d'avoir une idée objective des couts respectifs nour atteindre chacun de ces trois objectifs.

La politique du Ministère de la Qualité de la Vie se heurte toujours à cette difficulté de chiffrer ce qui est souvent inchiffrable: la qualité. Il y a des domaines où l'on peut chiffrer les bénéfices obtenus parce qu'on peut mettre en regard le coût de l'installation d'épuration d'une usine et le coût d'une installation qui aurait été nécessaire en aval pour permettre une nouvelle utilisation de l'eau. Mais il y a des cas où l'on n'y parvient pas.

Le Ministère de la Qualité de la Vie est très conscient de l'importance de tels calculs économiques mais aussi de leurs limites.

M. Gros apporte les précisions, ci-après:

En ce qui concerne ses interventions dans l'industrie papetière, l'Agence Rhin-Meuse a suivi une politique systématique d'aide aux opérations de réorganisation des circuits et de mise en place d'installations permettant d'économiser la pollution et l'eau. La station d'épuration est considérée comme la dernière étape de l'antipollution (que lon peut parfois éviter en papeterie en passant "en circuit fermé »).

Mais ces aides aux aménagements internes sont limités par le montant subventionnable calculé par l'Agence à partir de la pollution supprimée par les travaux considérés.

A une question de $M$. Serpaud sur la généralisation de l'emploi par les diverses Agences de la formule citée par M. DanIEL, M. le Président répond :

Les Agences de Bassin sont encore jeunes et chaque Agence a essayé d'élaborer une formule. Actuellement, on commence à ressentir le besoin d'uniformiser les différentes formules proposées. Il y a une discussion en cours entre les six Agences de Bassin sous l'égide de la Direction de la Prévention des Pollutions - non pas au titre de la tutelle, mais de l'harmonisation - pour essayer de trouver la moins mauvaise des formules.

Si les milieux extérieurs aux Agences ont des idées sur les formules à utiliser, nous accepterions toute suggestion à ce sujet; il suffirai qu'ils se rapprochent d'une des Agences de Bassin, ou de nous, pou que leurs propositions entrent comme éléments dans la discussion.

De façon plus générale, avant toute réalisation, l'industriel devrait prendre contact avec le Ministère de la Qualité de la Vie - tuteu réglementaire des Agences de Bassin - avec le Service des Mine. local et avec l'Agence de Bassin intéressée.

Pour M. BANAL, les aides de l'Etat dont il vient d'être question sont très utiles à titre d'incitation et dans la période de démarrage de l'action anti-pollution. Il serait fâcheux de donner l'impression que \& toute l'industrie va être subventionnée pour ne pas polluer». 
Vous avez parfaitement raison, répond M. le Président. D'ailleurs, les établissements nouveaux, les nouveaux outils de production ne font pas l'objet de subvention. Les Agences de Bassin peuvent seulement, dans ce cas, accorder des prêts.

M. le Président tire de la séance la «conclusion» en quatre points, ci-après :

$1^{\circ}$ On a bcaucoup parlé des problèmes de recyclage et j'ai noté qu'on avait peu parlé de l'action réglementaire, parce que les exposés ont été présentés par les Agences de Bassin. Il faudrait y revenii pour dire que cette action est très difficile car il est malaisé de fixer des objectifs aux industriels en matic̀re d'cau recyclée. 11 est possible de leur domner des objectifs en ce qui concerne la pollution des eaux... des objectifs de pollution à ne pas dépasser; mais, en ce qui concerne le volume d'eau à recycler, le problème est plus délicat.

Pour ces recyclages, une action incitative efficace du type de ceilo des Agences de Bassin, combinée avec la réglementation, est une bonne chose. C'est ce que l'on a fait par des programmes qui ont été à peine évoqués ce matin; ces programmes constituent les « contrats de branche » ou les «programmes de branche» qui ont pour objet la réduction des pollutions des usines existantes; ils sont assortis do modalités de financement et, dans le cas des contrats de branche, d'une participation de l'Etat.

$2^{\circ} \mathrm{La}$ diminution des quantités d'eau utiisées par les industries suppose des études techniques et économiques assez compliquées. Nous avons essayé de faire des études techniques générales; nous avons pu le faire sur quelques branches. J'avais tenté, il y a déjà quelques années, de faire un panorama de l'élasticité des diverses industries au regard des problèmes de l'eaul je dois dire que cela a été un fiasco. On n'en est pas sorti et on s'est rendu compte, comme le disait M. BaNAL, qu'on est obligé d'étudier chaque cas particulier.

En écoutant les exposés, j'ai constaté qu'il n'y a pas une usine avec son rejel et son problème d'épuration, mais une usine avec des circuits d'eau et des épurations spécifiques, qui, dans chaque cas, nécessitent des traitements particuliers. Donc, on atomise un peu le problème, ce qui le rend particulièrement difficile à résoudre. Les bureaux d'ingénieurs-conseils, ou même les spécialistes de l'usine, doivent étudier chaque cas; cela pose des problèmes, je le sais, aux bureaux d'ingénieurs-conseils, d'engineering.

3" Dans les calculs économiques, se pose le problème de savoir jusqu'où il faut aller pour imposer aux usines des recyclages d'eau. Nous avons évoqué les difficultés des calculs économiques afférents à ce genre de problème.

4" Je m'occupe de ces problèmes depuis 1966; au début, nous étions une poignée, mantenant nous sommes beaucoup plus nombreux. On en arrive à constater la nécessité d'un progrès équivalent dans les techniques de production et dans celles d'épuration. C'est une affirmation que j'avais posée il y a quelques années et qui n'avait pas paru évidente; je n'osais pas, à l'époque, dire le fond de ma pensée, à savoir qu'il fallait que les techniques de recyclage des eaux d'économic d'can et d'épuration aillent plus vite que les techniques de production dans la mesure où l'on avait un très important retard à rattraper. Ce que font les Agences de Bassin actuellement, c'est du rattrapage presque essentiellement...

J'ai l'impression que le souci des économies d'eau commence tout de même à pénétrer les milicux industriels: les exemples que nous avons entendu citer ce matin, nous n'aurions pas pu les citer il y a cinq ans. ll y a un mouvement qui est créé incontestablement, et je pense que ce mouvement est certainement dù à l'action des Agences de Bassin - peutêtre un peu aussi à l'action du Ministère de i'Enviromement - mais également à la diffusion des connaissances qui est réalisée grâce à des organismes comme la Société Hydrotechnique de France.

En terminant, je voudrais remercier la Société Hydrotechnique de France d'avoir permis cet échange de vues, car je crois que ce genre de rencontre est capital pour que des idées, comme celles que nous venons d'évoquer ce matin, puissent se diffuser et avoir droit de cité; or cela me paraît essentiel pour la politique de l'eau...

\section{Abstract \\ Recycling of water in paper mills}

It can be considered a general rule that the first step toward effective pollution control in factories is to redesign the wate circuits. Paper mills are a striking example of this, as circuit modifications usually result in more efficient use of raw materials and rationalized production.

Fifteen pollution control policies now being implemented in the Rhine-Meuse river valleys are based on action to be carried out in two phases. The first phase is to consist in redesigning the water supply and sewer systems with a view to maximal recyoling. In the second phase, non-recycled water will be purified at an appropriate station. In some cases, cspecially board mills, the purification plant will also be acting as the overall pulp recovery unit for the mills.

The sludge is returned to the pulper, whilst the water is generally processed and recycled for further use in the factory.

The Béghin Company's Kaysersberg board mills for example, which produce 200 metric tons of cardboard from waste paper daily, used to consume some $620 \mathrm{~m}^{3}$ of water hourly in 1972 .

The waste water passed through a settling tank and then discharged into a river. When the settling tank was operating properly, the residual load was $50 \mathrm{mg} / 1$ suspended matter and $150 \mathrm{mg} / \mathrm{l}$ COD. Breakdowns were frequent. The following comprehensive programme has since been put into effect with the regional finance authority's assistance:-

-Improvement of the settling and flocculation tank;
- Recycling of sludge (a daily raw material saving of 15 metric tons);

-Recycling of white water;

-Recycling of clear water after passing through a sand filter and chlorination.

The following results have been obtained to date:-

\begin{tabular}{|c|c|c|c|c|}
\hline & $\begin{array}{c}\text { WATER } \\
\left(\mathrm{m}^{3}\right)\end{array}$ & $\begin{array}{c}\mathrm{SS} * \\
(\mathrm{~kg} / \mathrm{mt} * \%)\end{array}$ & $\begin{array}{c}\text { BOD } \\
(\mathrm{kg} / \mathrm{mt})\end{array}$ & $\begin{array}{c}\text { COD } \\
(\mathrm{kg} / \mathrm{mt})\end{array}$ \\
\hline \multirow{2}{*}{1970} & 89 & 3.3 & 5.3 & 15.5 \\
Jate 1974 & 18.5 & 0.18 & 1.16 & 7.5 \\
\hline & & & & \\
\hline
\end{tabular}

Further improvements are expected. At the final stage, total bleed discharge should not exceed $9 \mathrm{~m}^{3}$ of water per metric ton produced. 